\title{
Der Rede wert
}

\section{Personalisierte Medizin - das können Naturheilverfahren auch!}

\author{
Die Begriffe personalisierte oder individualisierte Medizin werden \\ zunehmend für eine maßgeschneiderte Pharmakotherapie verwendet. \\ Die ursprüngliche Bedeutung beschreibt jedochein Vorgehen, das \\ Umfassenderes in die Behandlung einbezieht.
}

$\mathrm{P}$ ersonare, aus dem Lateinischen, bedeutet hindurchtönen. Persona bezeichnete ursprünglich eine im antiken griechischen Theater von den Schauspielern verwendete Maske, welche die Rolle des Schauspielers symbolisierte. Mit der personalisierten Medizin ist zunächst gemeint, dass jeder $\mathrm{Pa}$ tient unter weitgehender Einbeziehung individueller Gegebenheiten, also als Individuum, und über die reine Krankheitsdiagnose hinaus, behandelt wird. Verwendet wird der Begriff allerdings zunehmend sehr konkret für eine individualisierte Pharmakotherapie, die zusätzlich zu der konkreten Krankheitsdiagnose individuelle molekularbiologische Konstellationen berücksichtigt. Diesbezüglich hat es insbesondere in der Onkologie bahnbrechende Neuerungen gegeben.

\section{Die personale Seite im Blick}

Halten wir uns an die Begrifflichkeit im eigentlichen Sinne, beschreibt personalisierte oder individualisierte Medizin das Postulat und den Wunsch, die personale Seite des Menschen, also über seine Krankheit hinaus seine Persona, sein ganzes Wesen, zu therapieren. Eine Medizin also, die auch sonstige Beschwerden und Krankheiten sowie seelische und sozio-kulturelle Aspekte des Krankseins mit in ihr Bemühen um Heilung einbezieht. Dies ist ganz sicher der Wunsch unserer Patientinnen, die in ihrer ganzen Situation gesehen sein möchten, mit all dem, was die Krankheit für sie bedeutet, was sie krank gemacht hat, um so Wege zu finden, gesund zu werden und gesund zu bleiben. Gesundheit ist in diesem Sinne auch von der Weltgesundheitsorganisation WHO definiert worden und in deren Konstitution 1948 beschrieben: „Gesundheit ist ein $\mathrm{Zu}$ stand völligen körperlichen, seelischen und sozialen Wohlbefindens und nicht nur das Freisein von Krankheit und Gebrechen."

Intensive Zuwendung als Grundstein der Naturheilkunde

Die zunehmende Einbeziehung von Wissen aus der Psychosomatik, wie zum Beispiel in allen Brustzentren im Rahmen der psychoonkologischen Betreuung üblich, in unser alltägliches medizinisches Arbeiten ist in diesem Sinne zu begrüßen, und wird immer wieder von den Brustkrebs-Patientinnen als sehr bereichernd erlebt: „Ich war da einfach gut aufgehoben."

Und diesem Wunsch der Patientin, und auch des Arztes, nach personalisierter Medizin werden auch und gerade die Naturheilverfahren gerecht. Eine naturheilkundliche Anamnese beschränkt sich nie allein auf die aktuellen Beschwerden oder eine auswärts getätigte medizinische Diagnose, sondern umfasst immer sämtliche Symptome und Befindlichkeiten des Patienten, seine Krankheits- und Lebensgeschichte, seine Familiengeschichte, sein soziales und kulturelles Umfeld sowie seine Lebensgewohnheiten. Kritiker sehen gerade in dieser intensiven Zuwendung den hauptsächlichen Grund für die oft verblüffenden Erfolge naturheilkundlichen Arbeitens und verweisen auf den Placebo-Effekt. Das sei dahingestellt und gerne an anderer Stelle erörtert. Die Naturheil-

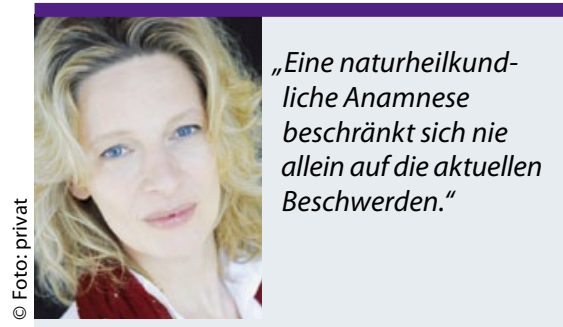

Dr. Anja Maria Engelsing,

Fachärztin für Frauenheilkunde, Homöopathie und Naturheilverfahren, Kirchgasse 3, 83075 Bad Feilnbach

verfahren sind eine sehr heterogene Gruppe von Therapieansätzen, die einzeln erläutert und hinsichtlich ihrer Wirkung validiert ohnehin getrennt betrachtet werden müssen.

Gerade bei vielen Krankheitszuständen in unserem Fachgebiet der Gynäkologie und Geburtshilfe, die durch gestörte hormonelle Regelkreisläufe oder intensiviertes Reagieren des Organismus auf physiologische Veränderungen beruhen, zum Beispiel Beschwerden der zweiten Zyklushälfte oder der Perimenopause, aber auch bei idiopathischer Dysmenorrhö junger Mädchen oder nicht konventionell therapiepflichtiger Hyperemesis gravidarum sind Naturheilverfahren auf jeden Fall einen Therapieversuch wert. Denn gerade diese ganzheitlichen Methoden leisten eine personalisierte Medizin zweifelsfrei, und sicher ist das in diesen sensiblen Zeiten im Leben einer Frau besonders gefragt.

Dr. med. Anja Engelsing 